\title{
Artgames vs. Game Art - Diskurse und Strategien der Computerspielkunst zwischen spielbarer Rhetorik und unspielbarer Ästhetik
}

\author{
Sebastian Möring ${ }^{1}$ \\ Angenommen: 15. Januar 2021 / Online publiziert: 8. Februar 2021 \\ (c) Der/die Autor(en) 2021
}

\section{Zusammenfassung}

Computerspiele sind vielfältig. Freizeitvergnügen, professioneller Sport, kulturbildend und kulturkritisch. Sie inspirieren die Kunst und mit ihnen wird Kunst gemacht. Dieser Beitrag betrachtet die Diskurse und Strategien der Computerspielkunst. Im Rückgriff auf die Game Studies und die medienwissenschaftliche Computerspielforschung beleuchtet er die Unterscheidung zwischen Artgames und Game Art (insbesondere Modifikationen) und versucht zu zeigen, dass beide Genres unterschiedlichen Diskursen entstammen, die mit verschiedenen Begriffen und künstlerischen Strategien operieren: Artgames setzen auf Spielbarkeit und Rhetorik und Game Art setzt auf Unspielbarkeit und Ästhetik. Sie grenzen sich so auf unterschiedliche Weise von den Sorgestrukturen kommerzieller Computerspiele ab.

\begin{abstract}
Computer games are diverse: recreational fun, professional sport, culture building, and culture critical. They inspire art, and art is made with them. This article examines the discourses and strategies of computer game art. Drawing on research from game studies and media studies, it examines the distinction between artgames and game art (especially modifications) and attempts to show that both genres originate from different discourses that operate with different concepts and artistic strategies: Artgames focus on playability and rhetoric, whereas game art focuses on unplayability and aesthetics. They distinguish themselves in different ways from the care structures of commercial computer games.
\end{abstract}

Der Spieleentwickler Rod Humble gestaltete das experimentelle Spiel The Marriage ([18]; Abb. 1), in dem es darum geht, das dynamische Modell einer grafisch abstrakt dargestellten - aufgrund der Farbkodierung aber dennoch als heteronormativ erkennbaren - Ehe am Laufen zu halten. Dies gelingt, wenn die Spielenden es schaffen, das Spiel mit der Maus so zu konfigurieren, dass weder das rosa noch das blaue Quadrat (links bzw. rechts, siehe Abb. 1) vollkommenen transparent werden oder so sehr schrumpfen, dass sie unsichtbar werden. Wenn die Spielenden den Mauszeiger auf eines der Quadrate halten, dann bewegen sich die sonst frei flotierenden Quadrate aufeinander zu und berühren sich. Diese als Kuss interpretierbare Berührung, macht die Quadrate opaker und lässt sie wachsen. Je länger sich die Quadrate nicht berühren desto eher schrumpfen sie und verlieren an Opazität. Humble hat mit dem Spiel sei-

Sebastian Möring

sebastian.moering@uni-potsdam.de

1 Universität Potsdam, Potsdam, Deutschland ne geschiedene Ehe verarbeitet und gibt zu Protokoll: „The Marriage is intended to be art" [20]. Es wurde in der Folge eines der bekanntesten Beispiele für Artgames.

Abb. 2 zeigt $S O D$ [22], eine Arbeit des niederländischbelgischen Medienkunstduos Jodi (Joan Heemskerk und Dirk Paesmans). Für dieses Werk wurde der 3D Ego-Shooter Wolfenstein $3 D$ [21] so modifiziert, dass seine dreidimensionale und gegenständliche Welt zu einer Schwarzweißgrafik abstrahiert wird, die an Barcodes und QR-Codes erinnert. Ungeübte Spieler*innen vermögen es kaum, die Wehrmachtssoldaten zu besiegen, gegen die man im Originalspiel in den Kampf zieht (für eine detaillierte Analyse von SOD siehe [38, S. 295-320]). Im Ausgangsspiel betrachtet man die dreidimensionale Spielwelt aus der Zentralperspektive und zielt mit dem Bildmittelpunkt auf Gegner, um diese zu eliminieren und so das Überleben der eigenen Spielfigur zu sichern und sie durch die labyrinthische Spielwelt zu navigieren. Das Spiel ist so nach Meinung einiger Autor*innen zu einem ,paradoxen Artefakt“" geworden [38, S. 295]. SOD ist eines der bekanntesten Beispiele für Game Art. 


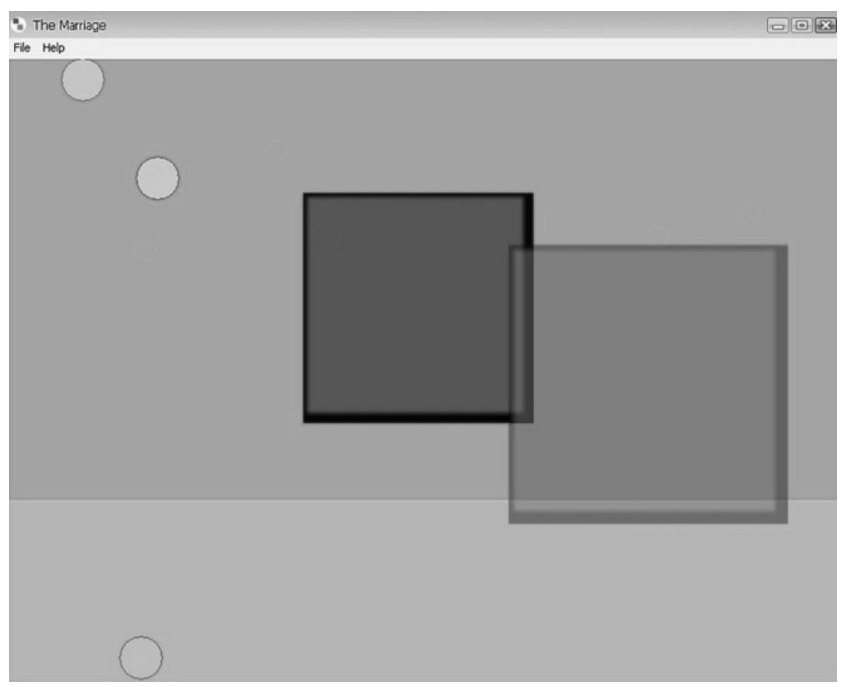

Abb. 1 The Marriage ([18], mit freundlicher Genehmigung des Designers)

Seit es Computerspiele gibt, wird versucht, die Existenz von Computerspielen zu legitimieren, indem man sie in den Rang der Kunst erhebt und so ihren kulturellen Wert herausstellt. So fragte etwa der Spieldesigner Ernest Adams in seinem gleichnamigen Essay „Will computer games ever be a legitimate art form?" und zählt im gleichen Atemzug einige arbiträre Aspekte von Computerspielen auf, die auf einen allgemein künstlerischen Charakter hinweisen [1]. Da Definitionen von Kunst aber weder einheitlich noch unveränderbar sind, raten andere Autor*innen dazu, die Frage nach Computerspielen als Kunst über Bord zu werfen: „Forget games, art doesn't have any sort of stable meaning in contemporary culture anyway“ [6, S. 9]. Der Kurator für Computerspielkunst, Matteo Bittanti, schlägt zugunsten eines breiten Computerspielkunstbegriffs vor
Game Art is any art in which digital games played a significant role in the creation, production, and/or display of the artwork. The resulting artwork can exist as a game, painting, photograph, sound, animation, video, performance or gallery installation $[3$, S. 9].

Hier unterstreicht Bittanti zum einen die Kunst als Schaffensprozess, in dem Computerspiele an irgendeiner Stelle eine wesentliche Rolle spielen, und zum anderen das Kunstwerk als Ergebnis eines solchen Prozesses, welches neben Computerspielen verschiedene andere mediale Formen annehmen kann. Dieser breite Kunstbegriff ist sinnvoll, um eine große Öffentlichkeit von der Vielfalt und Kreativität computerspielästhetischer Produktion zu überzeugen, aber er führt „zu erheblichen methodischen Problemen“, denn „Game Art [stellt sich, S.M.] als ein heterogenes Feld von Artefakten dar, deren künstlerische Strategien und Techniken, Mittel, Medien und Materialien breit gefächert sind" [38, S. 18]. Einem so breit gefassten Begriff entgehen Binnendifferenzierungen, die es ermöglichen, unterschiedliche Kunstgenres und sogar Diskurse innerhalb der Computerspielkultur abzubilden. Neben „Adaptionen und Aneignungen" vor allem visueller Charakteristiken von Computerspielen in anderen etablierten Kunstformen (vom Gemälde über die Fotografie bis zur Performance) und auf Computerspiele zurückgreifende „Ausdrucksformen wie Machinima und Demos" betont Schwingeler eine dritte Form der Computerspielkunst: „Modifikationen (kurz Mods) und eigenständige Produktionen von Computerspielen (so genannte Art Games) unter explizit künstlerischen Vorzeichen“ [38, S. 19].

Obwohl Schwingeler sich in seiner Arbeit ausschließlich auf Modifikationen konzentriert, spricht er in dem Zitat zwei Genres an, die sich im Laufe einer Diskursivierung
Abb. 2 SOD ([22], Screenshot aus [32])

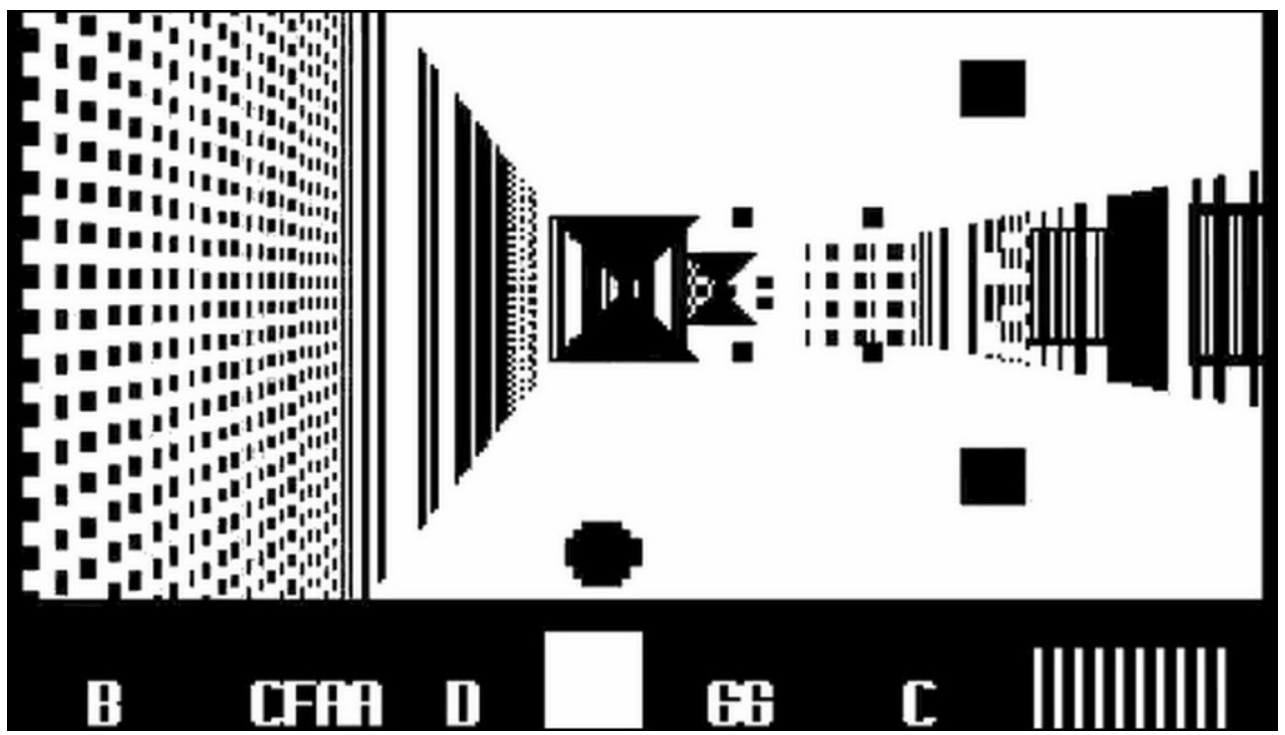


künstlerischer Praktiken im Zusammenhang mit Computerspielen herausgebildet haben. Dieser Beitrag nimmt deshalb speziell die Differenz zwischen Artgames und Game Art (insbesondere Mods) in den Blick. Er versucht zu zeigen, dass beide Genres unterschiedlichen Diskursen entstammen, die mit verschiedenen Begriffen und künstlerischen Strategien operieren. Im Folgenden wird gezeigt, Artgames setzen auf Spielbarkeit und Rhetorik und Game Art setzt auf Unspielbarkeit und Ästhetik.

\section{Games - Die Sorgestruktur kommerzieller Computerspiele}

Um zu einer Beschreibung der unterschiedlichen Strategien von Artgames und Game Art zu gelangen, bedarf es zuerst einer Beschreibung dessen, worauf sich beide Genres mit ihrer Andersartigkeit beziehen und vor dessen Hintergrund sie zu verstehen sind: kommerzielle Computerspiele. Vergleichbar mit der Kunst ist die Vielschichtigkeit von Computerspielen genauso wenig in einer einzigen Definition zu erfassen. Es haben sich dennoch Computerspielforscher*innen wie Jesper Juul darin versucht:

A game is a rule-based system with a variable and quantifiable outcome, where different outcomes are assigned different values, the player exerts effort in order to influence the outcome and, the player feels emotionally attached to the outcome, and the consequences of the activity are negotiable [23, S. 36].

Juuls populäre Definition klingt zunächst plausibel. Doch die Definition ergibt auch dann noch Sinn, wenn man aber die Begriffe „Game“ und „Player“ etwa durch „University“ und „Scholar“ oder durch „Supermarket" und „Client" ersetzt. Dies zeigt einerseits an, dass diese Definition zu weit ist und auch Systeme beschreibt, die keine Computerspiele sind. Andererseits gibt es Positionen, nach denen wir im Zeitalter der Ludifizierung von Kultur leben, in welchem kulturelle Systeme, die von Laien nicht als Spiel betrachtet werden, immer schon die Form von Spielen haben [13, 36]. Diese Idee schreibt die berühmte These des Kulturhistorikers Johan Huizinga, ,daß [sic!] menschliche Kultur im Spiel - als Spiel - aufkommt und sich entfaltet" [17, S. 7], in der heutigen Zeit unter den Bedingungen zunehmender Vernetzung und Digitalisierung fort. Vor diesem Hintergrund ist es dann wenig verwunderlich, dass Definitionen für Spiele auch andere kulturelle Systeme und Rituale beschreiben.

An Juuls Definition ist außerdem bemerkenswert, dass er Spielenden eine (emotionale) Verbundenheit mit dem Ergebnis eines Spiels zuschreibt. Dies wäre vielleicht der Punkt, an dem man am ehesten sagen würde, dass Juuls Definition eben nicht für den Supermarkt gilt, denn ob
Kund*innen emotional an ihren Einkaufsvorgängen hängen, ist unerheblich dafür, ob ein System als Supermarkt zu identifizieren ist. Doch diese Verbundenheit ist nicht nur emotionaler Natur. Sie ist grundlegender. Bei Computerspielen scheint es Juul wichtig, dass der Spielausgang die Spielenden kümmert. Dass er sie angeht. Alternativ hätte er an der Stelle seiner Definition auch schreiben können ,the player cares about the outcome." So oder so beschreibt dieser Aspekt der Definition einen emotionalen Bezug zum Spiel sowie eine grundlegende Motivation der Spielenden, überhaupt zu spielen. Es wird nur gespielt, wenn das Spielen die Spielenden kümmert, demnach heißt „,ein Spiel zu spielen ... eine Form von Sorge“" zu praktizieren [28, S. 290].

Diese Sorgestruktur von Computerspielen kann man leicht identifizieren, wenn man versucht $\mathrm{zu}$ beschreiben, worin die zentrale Aufgabe eines Computerspiels besteht bzw. worum man sich kümmern muss, um erfolgreich zu sein: Bei Tetris [34] kümmern sich die Spielenden darum, dass die Blöcke nicht den oberen Spielfeldrand berühren und das Spiel fortgesetzt werden kann. Bei Die Sims 4 [26] muss man sich ähnlich wie beim Tamagotchi [25] um die Befriedigung der existenziellen Bedürfnisse der zu betreuenden Spielfiguren kümmern. Spielen bedeutet demnach, sich um etwas zu sorgen und deshalb dafür $\mathrm{zu}$ sorgen, damit das, worum man sich sorgt, nicht eintritt. Wie die Definition von Computerspielen ist auch die Bedeutung des Begriffs „Spielbarkeit“ recht unscharf. Die hier beschriebene Sorgestruktur erlaubt es, genauer zu fassen, worum es geht, wenn im Folgenden von der Spielbarkeit und/oder ihrer Absenz die Rede ist.

\section{Artgames - Spielbarkeit und Rhetorik}

An den Ursprüngen der diskursiven Auseinandersetzung mit Artgames zeigt sich, dass anfangs kaum zwischen Artgames und Game Art unterschieden wurde. Das erklärt zum einen die breiten Definitionen, die es zum Thema aus dieser Zeit gibt, und gibt zum anderen der These Raum, dass sich seitdem neue Formen herausgebildet haben, die sich ästhetisch voneinander unterscheiden und deshalb unterschiedliche Bezeichnungen rechtfertigen. Schwingeler und Bittanti sehen die Anfänge der Artgames in Arbeiten wie The Night Journey (Abb. 3) des Medienkünstlers Bill Viola, das erstmals 2007 ausgestellt aber schon seit 1998 erdacht wurde ([38, Fußnote 20], [4]). Laut der Webseite findet das Spiel statt in einer ,mysterious landscape on which darkness is falling. There is no one path to take, no single goal to achieve, but the player's actions will reflect on themselves and the world, transforming and changing them both" [44]. Im Gegensatz $\mathrm{zu}$ dem oben beschriebenen Tetris verfügt The Night Journey über keine vorgegebene Sorgestruktur, die den Spielenden aufgibt, das Spiel vor dem Game Over 


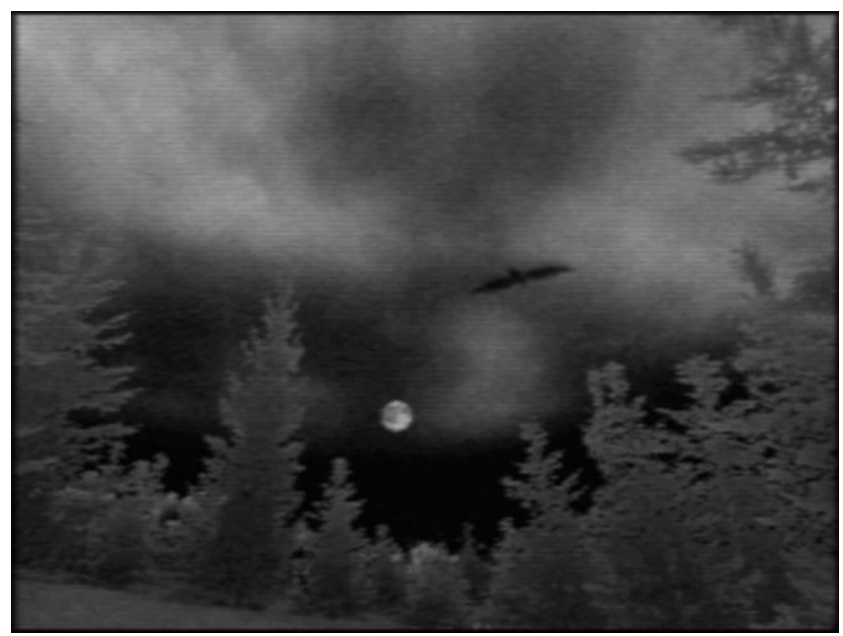

Abb. 3 The Night Journey ([44], Screenshot von der Webseite des Spiels)

zu bewahren. Stattdessen wird dem Spiel eine Vermittlungsabsicht eines bestimmten Themas oder Inhaltes bedacht. Den Macher*innen zufolge evoziert es ,,a sense of the archetypal journey of enlightenment through the mechanics of the game experience - i.e. the choices and actions of the player during the game" [44].

The Night Journey weist damit einige der Charakteristiken auf, welche Rebecca Cannon schon 2003 den Artgames zuschreibt:

Art Games may be made in a variety of media, sometimes from scratch without the use of a prior existing game. They always comprise an entire, (to some degree) playable game ... Art Games are always interactive - and that interactivity is based on the needs of competing ... Although both forms follow the lineage of Fine Art and computer games, Art Games explore the game format primarily as a new mode for structuring narrative, cultural critique. Challenges, levels and the central character are all employed as tools for exploring the game theme within the context of competition-based play (Cannon zitiert in [3, S. 8]).

Obwohl es zum Zeitpunkt dieser Beschreibung von Artgames noch keine Unterscheidung zwischen Game Art und Artgames gibt, nennt Cannon mit der Holistik und Spielbarkeit sowie der Innovation im Storytelling und dem Ausdruck von Kulturkritik wesentliche Merkmale, die später für die Charakterisierung von Artgames bestimmend werden. Die wettkampfbasierten Spielanforderungen gehen zugunsten der Kultur- und Medienkritik der Spiele verloren und ziehen sich hauptsächlich in die Domäne der MainstreamComputerspiele und der Independent Games zurück. Die mediale und materielle Vielfalt ist ein Merkmal, das später vor allem der Game Art zuzurechnen sein wird.

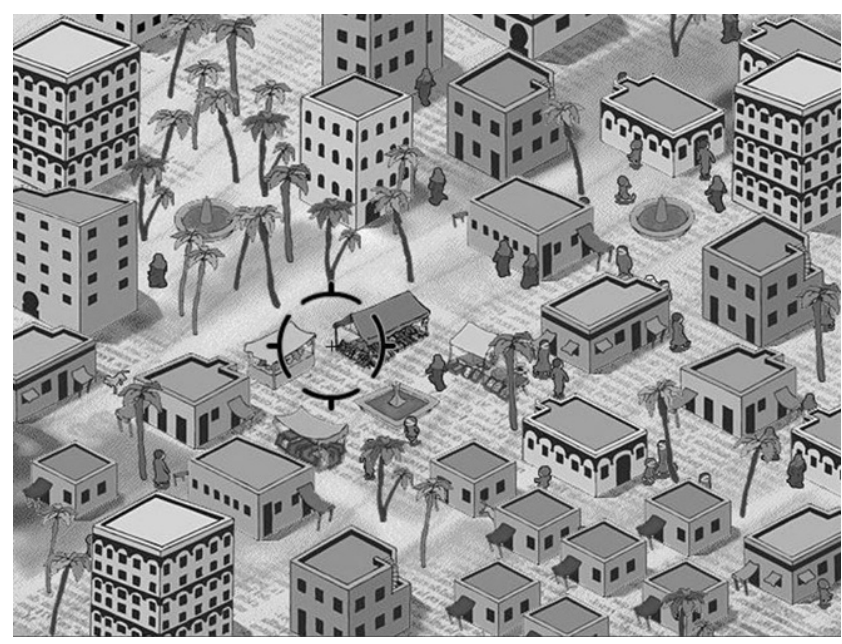

Abb. 4 September 12th ([11], mit freundlicher Genehmigung des Designers)

Etwa zur Mitte der 2000er-Jahre gesellt sich zu diesem vor allem aus der medien- und netzkünstlerischen Praxis heraus geführten Diskurs ein Diskurs aus den noch jungen interdisziplinären Game Studies. In dessen Rahmen werden parallel die Entwicklung von Artgames und die zugehörige Theorieproduktion betrieben. Beispielhaft hierfür steht September 12th ([11]; Abb. 4) des Spieldesigners und -theoretikers Gonzalo Frasca, in welchem die Spielenden aus einer Vogelperspektive auf eine hochgradig stereotypisierte mittelöstliche Stadt schauen, die zunächst hauptsächlich von Zivilist*innen bevölkert wird. Mithilfe eines Fadenkreuzes können sie auf Knopfdruck eine Bombe abwerfen. Wenn sie dies tun und dabei Zivilist*innen töten, verwandeln sich einige der Zivilist*innen nach einer Trauerphase in Terrorist*innen. Das Spiel verrät bereits offen im Eingangsbildschirm, dass es nicht gewonnen werden kann. Es kann aber auch nicht verloren werden. Dem Spiel fehlt eine Sorgestruktur oder anders gesagt, die Spielenden entscheiden, worum sie sich bei diesem Spiel sorgen wollen. Das Spiel selbst unternimmt keine Beurteilung der Leistungen der Spielenden in erfolgreich/erfolglos, gut/schlecht, sondern überlässt ihnen selbst diese Einordnung. Für Theoretiker*innen wie Olli Leino sind Spiele wie September 12th deshalb eher mit interaktiver Medienkunst zu vergleichen wie etwa Legible City von Jeffrey Shaw [40]. Leino zufolge ist die Möglichkeit des Scheiterns eine zentrale Eigenschaft von Computerspielen und ihre Abwesenheit ist eine zentrale Eigenschaft interaktiver Medienkunst [24].

Nichtsdestotrotz lassen sich in September 12th zwei der von Cannon genannten Charakteristiken finden: Es handelt sich um ein von Grund auf neu programmiertes und spielbares Spiel und es übt eine Form der Kulturkritik aus, indem es den kriegerischen Gegenschlag der USA als Folge des Attentats vom 11. September 2001 mit den Mitteln des Computerspiels problematisiert. Im frühen Artgame-Dis- 
kurs wird bereits zwischen politischen und ästhetischen Artgames unterschieden [41]. Politische Artgames verhandeln Themen wie Rassismus, Sexismus, Kapitalismus, Krankheit, Behinderung, soziale Ungleichheit, usf. Oft wird dabei die Bedeutung des Existenziellen in diesen Themen hervorgehoben. Kritiker argumentieren, dass Artgames vor allem deshalb besonders existenzielle Themen ausdrücken können, weil die Strukturen von Computerspielen, wie z.B. ihre Sorgestruktur, immer schon existenziell sind [30].

In Frascas Theoriearbeit werden Artgames wie September 12th als „games with a message“ [12] bezeichnet, ein Paradigma das später von Ian Bogost zur prozeduralen Rhetorik weiterentwickelt wird, die sogenannten persuasiven Spielen zu eigen ist. Dies bedeutet, dass Spiele nicht etwa sprachlich, sondern Kraft ihres interaktiven Regelwerks eine bestimmte mithin politische, persönliche usw. Aussage kommunizieren können. Obgleich man die Gültigkeit dieser Zuschreibung kritisieren kann (vgl. [28]), sind Bogost zufolge Spiele wie Rod Humble's The Marriage [18], Jonathan Blows Braid [5] sowie Jason Rohrer's Passage [37] als Artgames zu verstehen, die sich durch ,procedural rhetoric, introspection, abstraction, subjective representation, and strong authorship" auszeichnen [6, S. 13]. Dies bedeutet, dass Artgames einen subjektiven Blick auf ein oft persönliches Thema werfen und dies mit den Mitteln der Abstraktion und der prozeduralen Rhetorik umsetzen. Beispielhaft hierfür ist das eingangs beschriebene Spiel The Marriage, welches die Eheerfahrung seines Entwicklers visuell abstrakt und deshalb metaphorisch repräsentiert. Eine detaillierte metaphorische Analyse des Spiels [29] kann den Verdacht aber nicht ausräumen, dass Artgames vor allem deshalb Metaphorizität zugeschrieben wird, um ihre Kunstartigkeit zu markieren und tiefer liegende Bedeutungsebenen zu suggerieren. Dies geschieht möglicherweise aufgrund der oft mit dem Gebrauch von Metaphern assoziierten Annahme, es handele sich um besonders kreative und deshalb künstlerische Ausdrucksweisen [30]. Hinzu kommt, dass die drei von Bogost vorgestellten Artgame-Designer in Form von begleitenden Manifestos oder in Interviews den Anspruch artikulieren, dass ihre Spiele als Kunst verstanden und anerkannt werden [9, 19, 20]. Auf der Ebene der Spielstruktur aber ist The Marriage vergleichbar mit Tetris, denn es weist eine deutliche Sorgestruktur auf. Zum erfolgreichen Spielen des Spiels, was auf der Bedeutungsebene als vergleichbar mit einer erfolgreichen Partnerschaft gelesen werden könnte, kann man dessen metaphorische Bedeutung schlicht ignorieren. Es reicht zu wissen, welche Handlungen es braucht, um das Spiel so zu konfigurieren, dass die Quadrate weder bis zur Unsichtbarkeit ausbleichen noch schrumpfen. Wenn dies geschieht, ist das Spiel beendet analog zu Tetris, wenn die Blöcke den oberen Bildschirmrand berühren. Kritiker*innen wie John Sharp werfen der Artgames-Bewegung vor, einen konservativen und unzeitgemäßen Kunstbegriff sowie konservative Werte zu vertreten [39, S. 53-54]. Sie betonen auch, dass die Artgame-Bewegung nur einen kurzen Zeitraum füllt, bis etwa 2010 [39, S. 49]. Die dargestellten Strategien tauchten zwar zunächst in Artgames auf, finden aber in der Folge Eingang in die Independent Games und den Mainstream.

Am Beispiel von Artgames wie The Marriage, The Night Journey und September 12th ist festzuhalten, dass es sich dabei um Spiele mit und ohne Sorgestruktur handeln kann. Artgames wie The Night Journey und September 12th erlegen ihren Spieler*innen keine Sorgestruktur auf. Das heißt die Spielenden sind nicht gezwungen, bestimmte Aufgaben zu erfüllen, um weiterhin das Spiel spielen zu können. Stattdessen können sie selbst im Rahmen der interaktiven Möglichkeiten entscheiden, wie sie mit dem Spielobjekt umgehen wollen und worauf sie ihre Sorge richten wollen. Die fehlende Sorgestruktur ist mittlerweile zu einem zentralen Merkmal von Independent Games geworden. Independent Games wie Dear Esther [42], Gone Home [43], Firewatch [7], Walden, A Game [14] und Eastshade [10] werden aufgrund ihrer fehlenden Sorgestruktur oft als Walkingsimulatoren bezeichnet, denn in den Welten dieser Spieler führen Spielende ihre Spielfigur eher spazieren, als dass sie für deren Wohlergehen sorgen müssen.

\section{Game Art - Unspielbarkeit und Ästhetik}

Mit Blick auf Bittantis Definition (siehe oben) lässt sich zwischen einem engen und einem breiten Game-Art-Begriff unterscheiden, aus dem Artgames bereits herausgerechnet sind. Der breite Begriff betrifft alle Kunstwerke, in deren Schaffensprozess Computerspiele eine wichtige Rolle gespielt haben. Die Ergebnisse können dann in verschiedenen medialen Formen vorliegen, etwa als Malerei, Fotografie, Film (vgl. Machinima), Skulptur, Performance usw. Dieser Begriff von „Game Art ist gattungs-, material-, medienund technikunabhängig“ [38, S. 18]. Der engere Game-ArtBegriff konzentriert sich dann hauptsächlich auf „künstlerische Computerspielmodifikationen“" [38, S. 19]. Durch Eingriffe auf der Ebene der Software und der Hardware werden bestehende Spiele verändert. Sie werden dadurch zum Material in einem künstlerischen Prozess, der die Spiele oft an den Rand der Spielbarkeit und sogar bis hin zur Unspielbarkeit führt (vgl. [38, S. 27]). Alexander Galloway schlussfolgert ,gameplay is often neglected to the point of disappearance in most artist game mods" [15, S. 108]. Eingriffe auf der Ebene der Spielregeln bzw. der Spielstruktur kommen hingegen selten vor (vgl. [15]).

Das eingangs vorgestellte $S O D$ ist eine Modifikation, bei welcher durch ungerichtete Interventionen (die Künstler*innen bezeichnen sich selbst als nicht besonders begabte Programmierer*innen) in den „Quellcode“ des Spiels ein 
„aleatorisches Moment“ erzeugt wird. Die visuelle Ebene des Spiels wird auf unvorhersehbare Weise derart verändert, dass die gegnerischen Figuren nicht mehr zu erkennen und zudem schwer von der Spielumgebung unterscheidbar sind [38, S. 307, 299]. Die Spielstruktur bleibt aber von diesen Eingriffen unberührt. Das Spiel ist nach wie vor ein zeitund raumkritisches Actionspiel ([16] siehe [35]), in dem die Spielfigur von feindlichen Entitäten bedroht wird und die Spieler*innen dafür sorgen müssen, dass ihre Spielfigur am Leben bleibt. Dies gelingt am besten, wenn die Spielenden die gegnerischen Spielfiguren eliminieren. In $S O D$ ist die Sorgestruktur von Wolfenstein $3 D$ also intakt. Jedoch ist das Unterfangen, sich um das Überleben der Spielfigur und damit um die Bedingung zum fortgesetzten Spielen zu kümmern, dadurch ungleich erschwert worden, dass die Gegner kaum auszumachen sind.

An $S O D$ zeigen sich damit einige der ästhetischen Strategien, mit denen Game Art nach Galloway in der Regel operiert. Kommerzielle Spiele versuchen meist die Illusion einer virtuellen Welt zu erzeugen und versuchen deshalb alles, was diese Illusion stören könnte, wie etwa das Maschinenwerk, im Backstagebereich dieser Illusionen (z.B. Game Engines oder die Programmierungen) zu verstecken. Kommerzielle Spiele zielen nach Galloway auf größtmögliche Transparenz, was hier kontraintuitiv nicht Aufklärung, sondern ein Verstecken bedeutet [15, S. 114-115]. Game Art zielt auf den gegenteiligen Effekt und stellt genau diesen „Apparat“ hinter den Spielen aus [15, S. 114]. Das Zeigen der Mechanismen hinter den Spielen ist eine medienreflexive Strategie, die ein mediales Selbstbewusstsein vorweist (vgl. negative Medientheorie in [27]). Sie wird von Schwingeler als Opazität bezeichnet [38]. In SOD wird tatsächlich eine ästhetische Störung eingebaut. Im Sinne einer Störung der Wahrnehmung (griech. aisthesis) wird erschwert, das Spiel so spielen zu können, wie es gedacht war. Stattdessen wird dadurch die Aufmerksamkeit auf die Bedingungen der Möglichkeit des Computerspielens gelenkt. Wenn die Spielenden die aggressiven gegnerischen Figuren nicht sehen, können sie sie nicht eliminieren und ihre Spielfigur stirbt zwangsläufig früher oder später. Es ist dann unmöglich, der Sorgestruktur des Spiels zu entsprechen.

Damit ist zugleich eine zweite von Galloways Strategien aufgerufen, der zufolge die Aufmerksamkeit der Spiele vom Gameplay bzw. der Spielstruktur abweicht und sich stattdessen auf die (gestörten) Bedingungen der Wahrnehmbarkeit der Spiele richtet. Weitere Beispiele wie Adam Killer [8], Super Mario Clouds [2] und Velvet Strike anführend, konstatiert Galloway: „All these examples show how, in countergaming [= Game Art, S.M.], aesthetic experimentation often trumps interactive gameplay" $[15$, S. 118]. Bei Extremfällen wie Super Mario Clouds, wurde die Cartridge des Ausgangsspiels, Super Mario Bros. [33], auf der Hardwareebene soweit verändert, dass nur noch der Himmel und

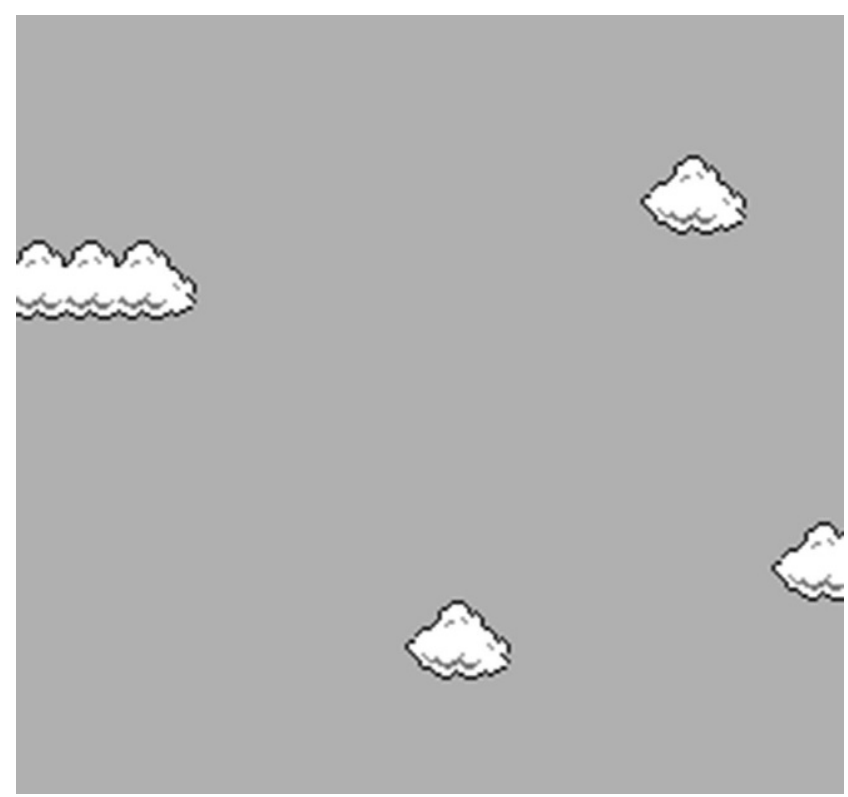

Abb. 5 Super Mario Clouds ([2], Screenshot des Autors)

die Wolken der Spielwelt zu sehen sind (Abb. 5). Ohne Spielfigur, gegnerische Entitäten, Dinge zum Einsammeln usw. ist die gesamte Sorgestruktur des Spiels ausgehebelt und die vermeintlichen Spieler*innen können nur dabei zusehen, wie die charakteristischen Wolken des Spiels vor blauem Himmel vorüberziehen.

Diese künstlerischen Strategien offenbaren einen wesentlichen Widerspruch der Game Art, aber sie zeigen auch, wo sich die Spielmodifikationen des engen Game-Art-Begriffs und Kunstwerke, die der weite Game-Art-Begriff umschreibt, annähern:

artist-made game mods will be able to resolve the essential contradiction of their existence thus far: that they have sought largely to remove their own gameplay and lapse back to other media entirely (animation, video, painting) [15, S. 126].

Spielmodifikationen wie Super Mario Clouds, obwohl sie immer noch als Software prozessiert werden, haben dementsprechend ästhetisch mehr Gemeinsamkeiten mit dem Medium des Films, da in ihnen die Sorgestruktur entfernt wurde. In der künstlerischen Computerspielfotografie ist die Sorgestruktur des fotografierten Spiels durch die Medialität der Fotografie ausgeschlossen, obwohl sie in der Gestaltung des Fotos durchaus eine Rolle spielt [31]. In beiden Fällen wird aber die Aufmerksamkeit weg von der Spielbarkeit hin zu den Bedingungen der Wahrnehmbarkeit von Computerspielen und damit auf die Grundlagen ihrer Ästhetik gelenkt. 


\section{Ausblick}

Weder Artgames noch Game Art erfüllen Galloway's Vision einer „radical action“ der Game Art, die er „Countergaming“" nennt, analog zu Godards „Countercinema“ [15, S. 126]. Er kritisiert, dass die Negation der Spielstruktur der Game Art die Aufmerksamkeit meist auf die Visualität von Computerspielen lenkt, aber nicht auf das, was sie ausmacht, die Spielstruktur. Galloway hofft auf eine Computerspielkunst, die die Spielstrukturen der Spiele selbst kritisiert und dies mithilfe von anderen kreativen Spielstrukturen. Bisher bleibt nur das Mittel der Negation, die im Wegfallen der Sorgestruktur (Artgames und breite Game Art) oder in der Verunmöglichung des Umgangs mit der Sorgestruktur besteht.

Funding Open Access funding enabled and organized by Projekt DEAL.

Open Access Dieser Artikel wird unter der Creative Commons Namensnennung 4.0 International Lizenz veröffentlicht, welche die Nutzung, Vervielfältigung, Bearbeitung, Verbreitung und Wiedergabe in jeglichem Medium und Format erlaubt, sofern Sie den/die ursprünglichen Autor(en) und die Quelle ordnungsgemäß nennen, einen Link zur Creative Commons Lizenz beifügen und angeben, ob Änderungen vorgenommen wurden.

Die in diesem Artikel enthaltenen Bilder und sonstiges Drittmaterial unterliegen ebenfalls der genannten Creative Commons Lizenz, sofern sich aus der Abbildungslegende nichts anderes ergibt. Sofern das betreffende Material nicht unter der genannten Creative Commons Lizenz steht und die betreffende Handlung nicht nach gesetzlichen Vorschriften erlaubt ist, ist für die oben aufgeführten Weiterverwendungen des Materials die Einwilligung des jeweiligen Rechteinhabers einzuholen.

Weitere Details zur Lizenz entnehmen Sie bitte der Lizenzinformation auf http://creativecommons.org/licenses/by/4.0/deed.de.

\section{Literatur}

1. Adams EW (2014) Will computer games ever be a legitimate art form? J Media Pract 7:67-77. https://doi.org/10.1386/jmpr.7.1.67/

2. Arcangel C (2002) Super Mario Clouds

3. Bittanti M (2006) Game art. (This is not) A manifesto. (This is) A disclaimer. In: Bittanti M, Quaranta D (Hrsg) Gamescenes: art in the age of videogames. Johan \& Levi, Milano, S 7-14

4. Bittanti M (2018) Art game: Bill Viola's the night journey (2018). In: GAMESCENES. https://www.gamescenes.org/2018/06/artgame-bill-violas-the-night-journey-2018.html. Zugegriffen: 23. Okt. 2020

5. Blow J (2008) Braid, [Xbox 360 et.al.] (Microsoft Game Studios)

6. Bogost I (2011) How to do things with videogames, Kindle Edition. University of Minnesota Press, Minneapolis

7. Campo Santo (2016) Firewatch, [PlayStation 4; Xbox One; Microsoft Windows; Mac OS; Linux] (Campo Santo, Panic)

8. Condon B (1999) Adam Killer

9. Dahlen C (2010) AVC at GDC '10: An interview with art-game creator Jason Rohrer. In: AV Club. http://www.avclub.com/articles/ avc-at-gdc-10-an-interview-with-artgame-creator-ja,39180/. Zugegriffen: 22. Nov. 2020
10. Eastshade Studios (2019) Eastshade, [Windows PC] (Eastshade Studios)

11. Frasca G (2003) September 12th, [Browsergame]

12. Frasca G (2007) Play the message. Play, game and videogame rhetoric. PhD Thesis (unpublished), IT University of Copenhagen

13. Frissen V, Lammes S, de Lange M, de Mul J, Raessens J (2015) Homo ludens 2.0: play, media, and identity. In: Frissen V, de Lange M, Raessens J, Lammes S, de Mul J (Hrsg) Playful identities: the ludification of digital media cultures. University Press, Amsterdam, S 9-50

14. Fullerton T, USC Game Innovation Lab (2017) Walden, a game, [Windows PC, MacOS] (USC Games)

15. Galloway A (2006) Countergaming. In: Gaming: essays on algorithmic culture. University of Minnesota Press, Minneapolis, S $107-126$

16. Günzel S (2012) Egoshooter: das Raumbild des Computerspiels. Campus, Frankfurt a.M.

17. Huizinga J (2004) Homo Ludens. Vom Ursprung der Kultur im Spiel, 19. Aufl. Rowohlt, Reinbek

18. Humble R (2006) The marriage, [Windows PC]

19. Humble R (2006) Game rules as Art. The Escapist. http://www. escapistmagazine.com/articles/view/video-games/issues/issue_41/ 247-Game-Rules-as-Art. Zugegriffen: 29. Jan. 2021

20. Humble R (2009) The marriage. http://www.rodvik.com/rodgames/ marriage.html. Zugegriffen: 22. Nov. 2020

21. id Software (1992) Wolfenstein 3D, [DOS] (Apogee Software)

22. Jodi (1999) SOD

23. Juul J (2005) Half-real. Video games between real rules and fictional worlds, kindle edition. MIT Press, Cambridge

24. Leino OT (2013) Playability and its absence-A post-ludological critique. In: Proceedings of DiGRA 2013: DeFragging Game Studies. Digital Games Research Association DiGRA

25. Maita A (1996) Tamagotchi (Bandai)

26. Maxis, The Sims Studio (2014) The Sims 4, [Microsoft Windows; Mac OS] (Electronic Arts)

27. Mersch D (2006) Medientheorien zur Einführung. Junius, Hamburg

28. Möring S (2013) Games and metaphor-A critical analysis of the metaphor discourse in game studies. IT University of Copenhagen, Copenhagen

29. Möring S (2015) Simulated metaphors of love. How the marriage applies metaphors to simulate a love relationship. In: Enevold J, MacCallum-Stewart E (Hrsg) Game love: essays on play and affection. McFarland \& Co, Jefferson, S 196-215

30. Möring S (2016) What is a metaphoric artgame?-A critical analysis of metaphor in the art game discourse and in artgames. In: Fahlenbrach K (Hrsg) Embodied metaphors in film, television, and video games: cognitive approaches. Routledge, London, S 269-284

31. Möring S, de Mutiis M (2019) Camera ludica: reflections on photography in video games. In: Fuchs M, Thoss J (Hrsg) Intermedia games-Games inter media: video games and intermediality. Bloomsbury Academic, New York, S 69-94. https://doi.org/10. 5040/9781501330520.ch-003

32. Myers A (2014) Case study \#3: fire in the hole-The obviously non-short history of art games. NMC media-N journal of the new media caucus. http://median.newmediacaucus.org/caa-edition/ case-study-3-fire-in-the-hole-the-obviously-non-short-historyof-art-games/. Zugegriffen: 24. Nov. 2020

33. Nintendo (1985) Super Mario Bros., [Nintendo Entertainment System] (Nintendo, Kyoto)

34. Pajitnov A, Gerasimov V, Pavlovsky D (1984) Tetris, [Various]

35. Pias C (2000) Computer Spiel Welten. PhD Thesis, Bauhaus University Weimar

36. Raessens J (2006) Playful identities, or the ludification of culture. Games Cult 1:52-57. https://doi.org/10.1177/1555412005281779

37. Rohrer J (2007) Passage, [Windows PC et.al] 
38. Schwingeler S (2014) Kunstwerk Computerspiel - digitale Spiele als künstlerisches Material: eine bildwissenschaftliche und medientheoretische Analyse. transcript, Bielefeld

39. Sharp J (2015) Works of game: on the aesthetics of games and art. MIT Press, Cambridge

40. Shaw J (1988) The legible city

41. Stalker PJ (2005) Gaming in art: a case study of two examples of the artistic appropriation of computer games and the mapping of historical trajectories of "art games" versus mainstream computer games (University of the Witwatersand)

42. The Chinese Room (2012) Dear Esther, [Windows] (The Chinese Room)

43. The Fulbright Company (2013) Gone home, [Windows] (The Fulbright Company)

44. USC Game Innovation Lab (2007) The night journey. https://www. thenightjourney.com. Zugegriffen: 23. Okt. 2020 